\title{
LES HÉMATOZOAIRES DES SERPENTS DE STANLEYVILLE
}

\author{
(Congo Belge)
}

\section{Par J. SCHWETZ}

De septembre 1927 à février 1930. nous avons examiné, au Laboratoire de Stanleyville, le sang de 356 serpents, provenant des environs plus ou moins immédiats du Laboratoire. La liste ci-jointe des espèces donne, en même temps, une idée assez exacte de leur fréquence relative à Stanleyville.

Nous devons la détermination de nos serpents à M. de Vitte, Conservateur au Musée du Congo Belge, à Tervueren (Bruxelles). Nous l'en remercions, ainsi que M. Schouteden, Directeur de ce Musée.

En réalité, notre collection de serpents examinés contient encore un certain nombre d'espèces ; mais comme il s'agit de spécimens uniques par espèce et négatifs au point de vue parasitologique, nous avons cru inutile de trop allonger notre liste. Disons aussi qu'il est possible qu'il se soit glissé des erreurs dans le nombre de spécimens des espèces affines et notamment dans le groupe de serpents gris. Mais tous les serpents parasités furent envoyés à part et déterminés avec précision.

Il résulte de cette liste que les serpents les plus communs de Stanleyville sont : la vipère grise (Causus rhombeatus) et les serpents gris (Holorupholis et Boodon olivaceus). Viennent ensuite: la vipère cornue (Bitis nasicornis) et le serpent vert (Chlorophis irregularis).

En ce qui concerne l'index parasitaire sanguin de nos serpents, on aura constaté son insignifiance aussi bien quantitative que qualitative. Non seulement - sur un si grand nombre d'animaux examinés - nous n'avons rien trouvé d'autre que des hémogrégarines, mais même ce dernier protozoaire ne fut rencontré que bien rarement. Ce résultat est surtout frappant en comparaison avec le résultat de l'examen des grenouillès (et même des crapauds) de Stanleyville, qui sont parasités dans une très forte proportion par plusieurs espèces de parasites sanguicoles (Schwetz, 1930). C'est ainsi que nous n'avons jamais trouvé un seul trypanosome chez plusieurs centaines de nos ophidiens !

En ce qui concerne les hémogrégarines, on voit, d'après notre Annales de Parasitologie, T. IX, $\mathrm{N}^{\circ} 4 .-1^{\mathrm{er}}$ juillet 1931, p. 303-310. 


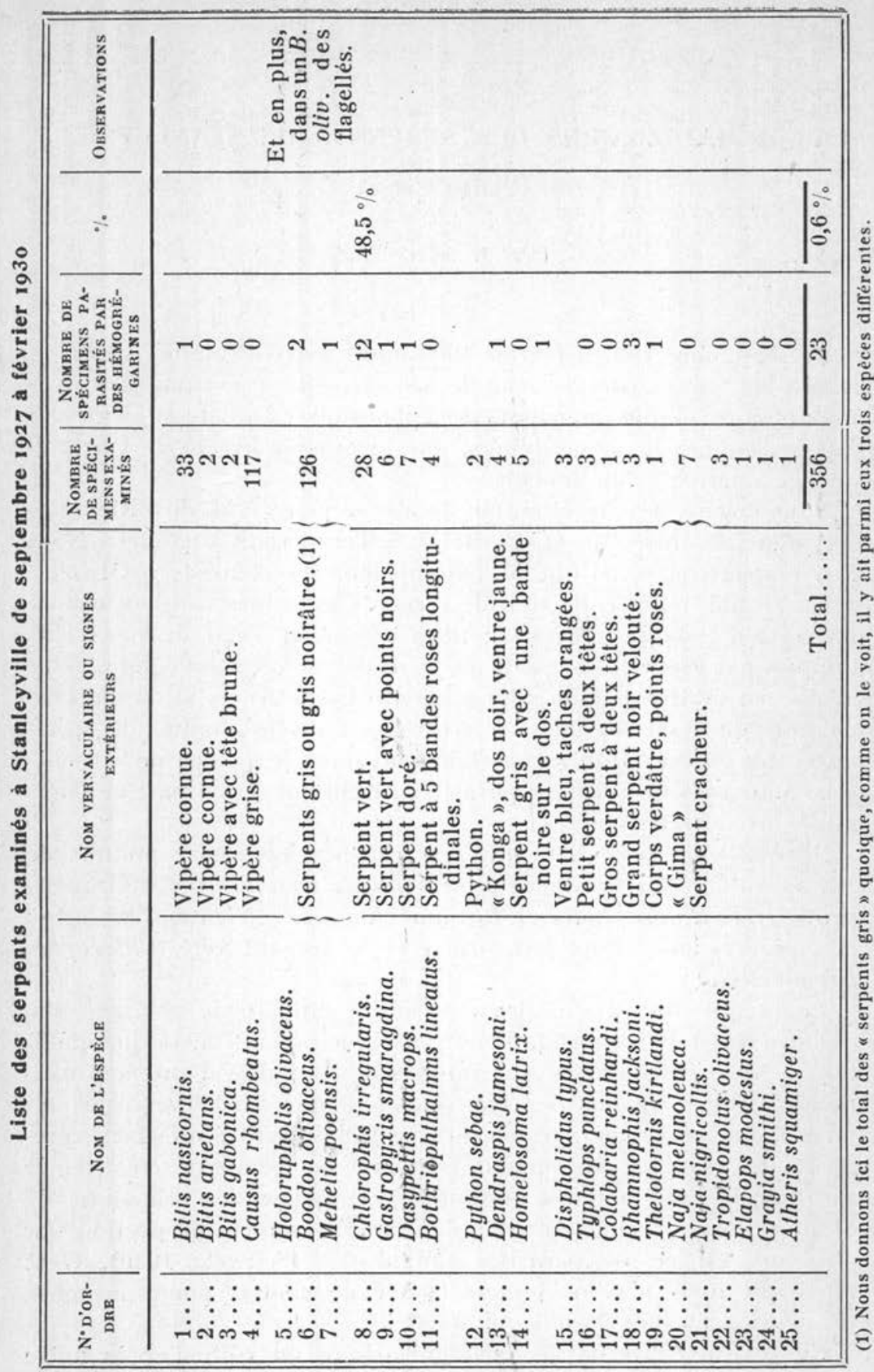


liste, que la proportion de serpents infestés par ce parasite est très variable suivant l'hôte. Ainsi, nous n'avons pas trouvé une seule hémogrégarine chez les 117 Causus rhombeatus et par contre, sur 28 Chlorophis irregularis, nous avons trouvé 12 infestés, soit près de la moitié. En général, les hémogrégarines étaient plutôt rares chez nos serpents et quelquefois il fallait examiner assez longtemps les frottis avant de trouver un globule rouge parasité. Ce n'est qu'une seule fois, chez un Mehelya poensis, que nous avons trouvé une très forte infection: 50 globules parasités, dont 4 avec des hémogrégarines doubles, sur 300 , soit 17 p. 100 de głobules infectés. Dans ce cas, nous avons même trouvé des globules rouges contenant 3 hémogrégarines.

En règle générale, les hémogrégarines de nos serpents étaient notablement plus petites que celles des grenouilles et crapauds (de même d'ailleurs que les globules rouges eux-mêmes), mais leur morphologie variait non seulement d'une espèce à l'autre, mais même d'un spécimen à l'autre de la même espèce. Bien plus, dans le même serpent et dans le même frottis, on trộvait quelquefois des hémogrégarines un peu différentes les unes des autres. Dans ces conditions, nous avons cru inutile de donner des mensurations, nécessairement trop vagues et même un peu arbitraires, et nous nous sommes borné à faire figurer les plus typiques des formes trouvées.

Dans la fig. 1, sont reproduites les hémogrégarines trouvées dans deux Chlorophis irregularis, serpent vert des arbres (A et B). Dans le premier serpent (A), on voit une hémogrégarine encapsulée, intra et extraglobulaire, ainsi que des spécimens extra-globulaires sans capsule (5) et des capsules vides (4). Les globules rouges parasités sont tantôt hypertrophiés (2 et 11) et tantôt déformés (15). L'hémoglobine est parfois altérée (16). A noter le curieux globule (17) où le parasite est sous le noyau. A noter également les curieuses formes extra-globulaires des numéros 19 et 15 . Dans le $\mathrm{n}^{\circ} 9$, le plissement de l'hémogrégarine a provoqué une segmentation de son noyau. Nous nous abstenons de l'interprétation de la curieuse formation intra-globulaire du $n^{\circ} 14$, qui ressemble un peu à un Dactylosoma, mais qui n'en est évidemment pas un. Dans le deuxième Chlorophis irregularis (B), on voit en somme la même hémogrégarine que dans le premier, mais sans capsule.

Dans A et B de la fig. 2, sont figurées les hémogrégarines trouvées dans deux spécimens de Rhamnophis jacksoni (grand serpent complètement noir et velouté). Dans le premier serpent (A), il s'agit en somme d'une hémogrégarine ordinaire, longue et relati-

Annales de Parasitologie, T. IX, No $4 .-1^{\text {er }}$ juillet 1931.

20. 
vement étroite, avec le noyau compact. Tandis que dans le deuxième serpent (B), il s'agit d'une hémogrégarine vraiment spéciale, peu ordinaire, aussi bien par sa forme générale que par son noyau. Le

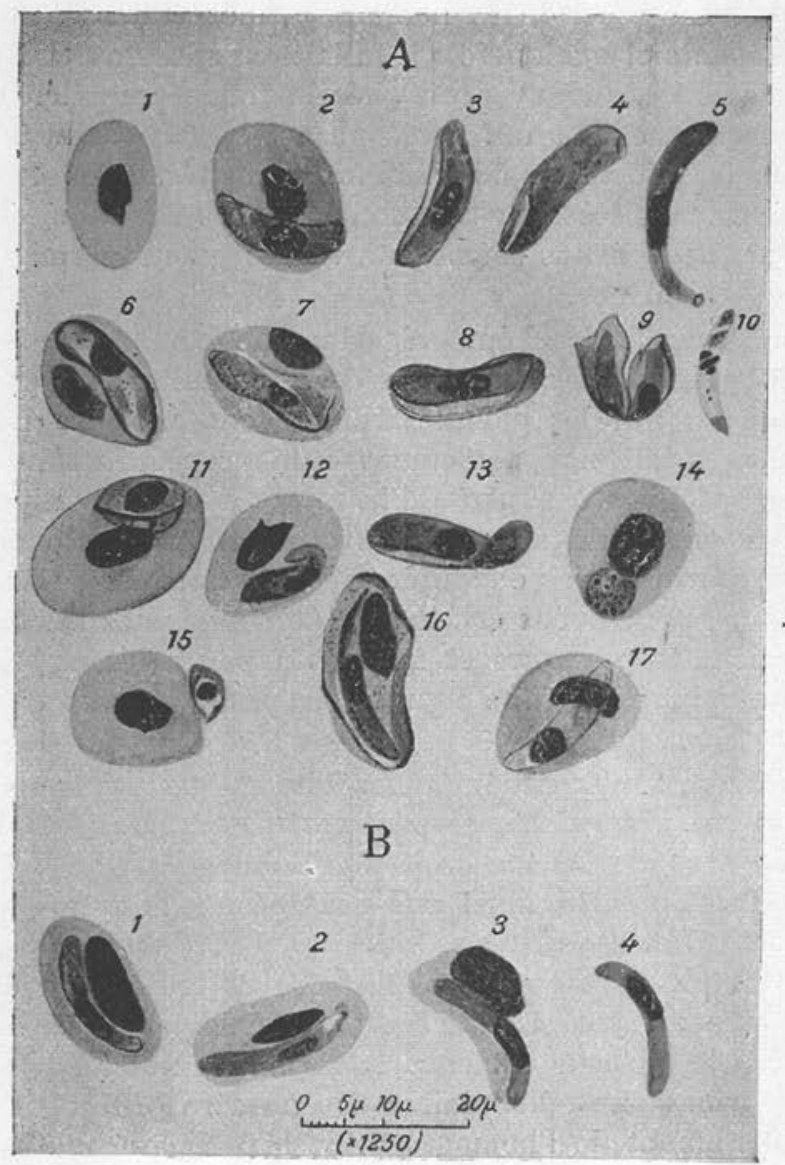

FıG. 1. - Chlorophis irregularis.

parasite est, avec des variations suivant le stade de son évolution, assez trapu, parfois aussi large que long. Le protoplasme est pâle et le noyau se présente sous la forme d'une bande transversale peu foncée. Toutefois, dans le $\mathrm{n}^{\circ} 3$, le noyau est compact.

Dans C, de la même fig. 2, sont reproduites les quelques hémogrégarines extrêmement rares trouvées dans le sang d'une vieille 
et énorme vipère cornue (Bitis nasicornis), seul spécimen parasité sur les 33 examinés. Les trois parasites trouvés dans ce serpent sont un peu différents les uns des autres. Les deux formes libres

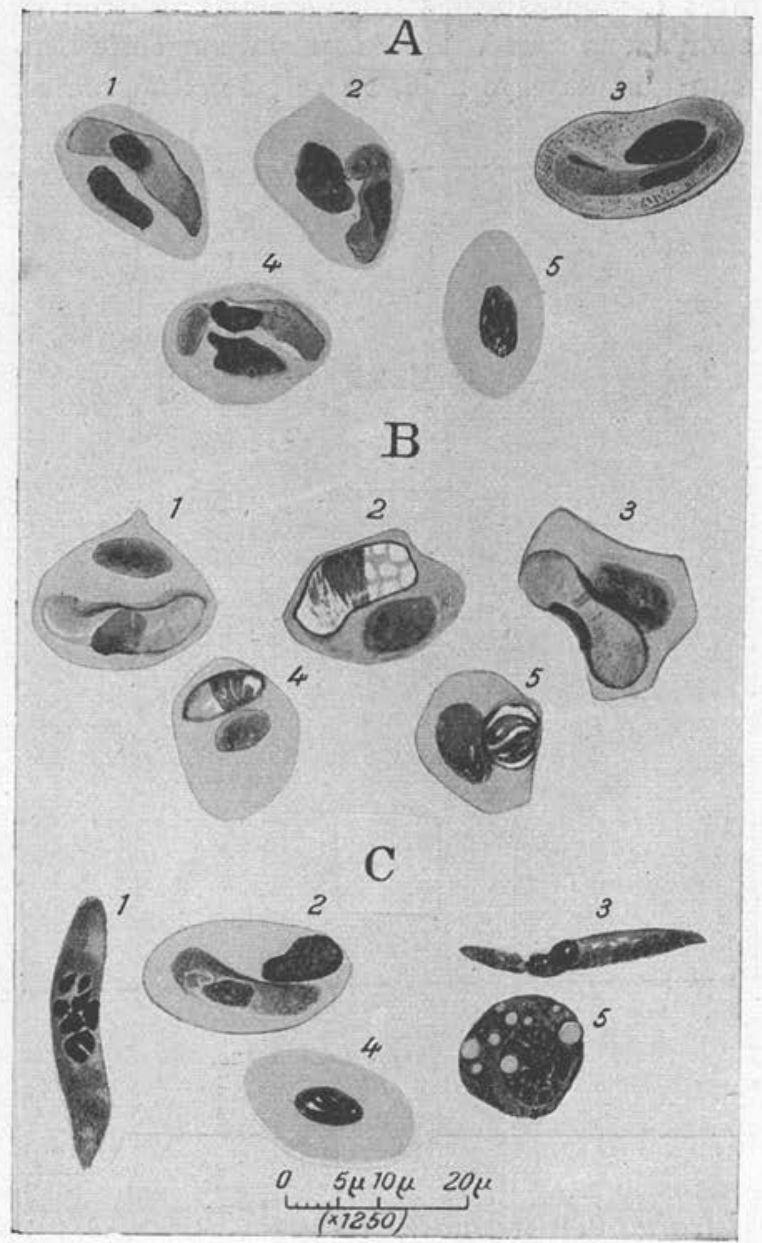

Fig. 2. - A. B. Rhamnophis jacksoni. C. Bitis nasicornis.

(1 et 3 ) sont beaucoup plus longues que la seule forme intraglobulaire (2). Et tandis que, dans les numéros 2 et 3 , le noyau est compact, il est très segmenté dans le $\mathrm{n}^{\circ} 1$ où il se présente sous la forme de 8 grosses masses nucléaires. Le $n^{\circ} 5$ reproduit un leuco- 
cyte spécial, assez commun d'ailleurs dans le sang des ophidiens, surtout dans certains genres et espèces.

La fig. 3 reproduit les parasites trouvés dans le sang d'un serpent gris noirâtre (Boodon olivaceus). En A, sont reproduites quelques rares hémogrégarines intra-globulaires trouvées chez cet animal. Nous n'avons rien à dire de spécial sur cette hémogrégarine ordinaire, sauf que, dans le $n^{\circ} 4$, on voit deux opercules, un à cha-

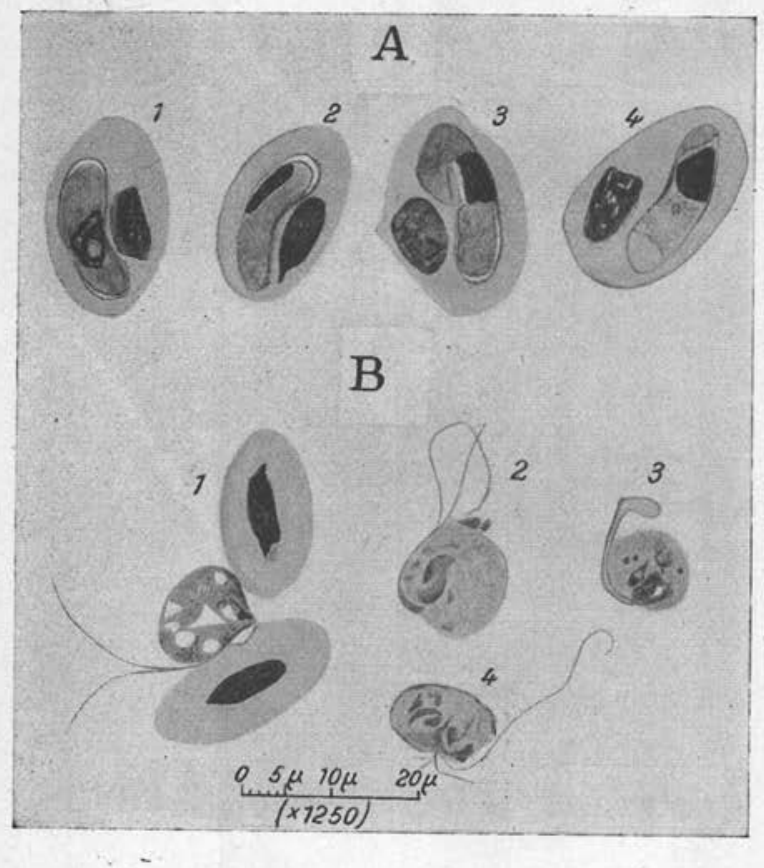

FIG. 3. - Boodon olivaceus.

que extrémité. En B, nous avons reproduit 4 spécimens d'un flagellé trouvé dans le sang de ce même serpent. Nous disons « dans le sang », entre guillemets, parce qu'il est plus que probable que ce flagellé a pénétré dans le sang, c'est-à-dire sur les lames, au moment de la confection des préparations. Comme nous prenions habituellement le sang dans le cœur, il est probable que nous avons touché la cavité générale ou même un organe digestif. A frais, ce flagellé pullulait dans le sang et se déplaçait si vite qu'il était difficile d'observer sa forme. Dans les frottis colorés, nous n'avons plus rien trouvé, sauf de-ci, de-là, de petites taches rosées. Ce n'est 
qu'au bord des frottis que nous avons fini par retrouver quelques rarissimes flagellés plus ou moins bien colorés et reproduits en B, fig. 3 .

Plusieurs auteurs ont trouvé divers flagellés dans le sang des animaux à sang froid (voir Wenyon, Protozoology, p. 271). Parmi ces observations, nous en citerons deux.

Plimmer a trouvé des Trichomonas dans le sang de plusieurs serpents du Jardin Zoologique de Londres. Mais il s'agissait d'ophidiens morts. Plus probante est l'observation de Lavier et Galliard, qui trouvèrent dans le sang de la patte d'un Bufo calamita un grand nombre de flagellés du genre Hexamitus. Et ce qui rend cette observation encore plus intéressante, c'est le fait que l'intestin de ce crapaud ne présentait pas d'Hexamitus.

Comme nous venons de le dire, chez nous il s'agissait de sang pris au cœur d'un serpent vivant. Le contenu intestinal n'a pas été examiné, de sorte que la provenance de notre flagellé reste douteuse. Sa détermination exacte reste également douteuse. Il ne s'agit évidemment pas d'un Hexamitus qui a six flagelles. Notre flagellé, avec ses trois flagelles, ressemble à un Trichomonas, mais il n'a pas, ou l'on n'y voit pas, d'axostyle. Il est vrai que nous n'avons pas fait une fixation ni une coloration spéciales permettant de voir les détails du parasite.

Dans notre travail, nous nous sommes borné à examiner le sang proprement dit, sans chercher systématiquement les formes de reproduction de nos hémogrégarines dans les organes internes. A plusieurs reprises, nous avons fait des frottis de poumon, de foie et de rate, mais nous y avons trouvé les mêmes hémogrégarines que dans le sang, c'est-à-dire soit des formes intra-globulaires, soit des formes libres.

Nous venons de voir que les hémogrégarines trouvées par nous chez divers ophidiens de Stanleyville sont un peu différentes, suivant le genre ou l'espèce de l'hôte et même suivant les spécimens de la même espèce. Bien plus, chez le même serpent, nous avons trouvé différentes hémogrégarines, ce qui provient, probablement et en partie du moins, de la différence du stade de l'évolution de l'hématozoaire.

S'agit-il de plusieurs espèces ? Et si oui, faut-il le's nommer et, dans l'affirmative, comment les nommer? D'après le nom de leur hôte, du serpent? Dans le doute, nous avons préféré nous abstenir plutôt que d'encombrer la terminologie, sans une base solide.

Comme le dit le professeur Brumpt (1928) : «Une classification rigoureuse des hémogrégarines ne pourra être faite que le jour où l'infection expérimentale d'hôtes différents par une espèce déter- 
minée pourra être effectuée. Alors seulement il sera possible de déterminer la valeur de la spécificité biologique admise actuellement et d'indiquer de meilleurs critères spécifiques. »

Comme on a incriminé les acariens, les tiques et les porocéphales dans la transmission des hémogrégarines des reptiles (Wenyon, 1926), nous croyons utile de signaler que nos serpents étaient parasités dans une forte proportion par des porocéphales. Chose curieuse: ce sont surtout la vipère cornue et la vipère grise (si peu infectées par les hémogrégarines), qui sont très parasitées par les porocéphales. En ce qui concerne les tiques, nous avons trouvé à plusieurs reprises quelques rarissimes A ponomma (A. exornatum et $A$. laeve) sur la vipère grise (Causus rhombeatus) et le serpent gris (Boodon olivaceus). Ces deux arachnides (les tiques et les porocéphales) ne semblent donc pas devoir jouer un rôle dans la transmission des hémogrégarines des ophidiens.

\section{BibLIOGRAPHIE}

Brumpt (E.). - Hémogrégarine (Hamogregarina lahillei n. sp). d'une couleuvre à collier (Tropidonotus natrix) de Corse. Annales de Parasitologie, VI, 1928, p. 23-26.

Foley (H.) et Catanei (A.). - L’hémogrégarine de la vipère à cornes (Hamogregarina seurati Laveran et Petlit, 1911); ses formes de multiplication. Bull. Soc. pathol. exot., XVIII, 1925, p. 393-397.

- Hémogrégarines de trois couleuvres et d'un lézard d'Algérie. Arch. Institut Pasteur d'Algérie, V, 1927, p. 109-119, 4 pl.

Laveran et Petrit. - Contribution à l'étude des hémogrégarines de quelques sauriens d'Afrique. Bull. Soc. pathol. exotique, II, 1909, p. 506-514.

Lavier (G.) et Galliard (H.). - Parasitisme sanguin d'un Hexamilus chez un crapaud Bubo calamita. Ann. de Parasitologie, III, 1925, p. 113-115.

Schwetz (J.). - Notes protozoologiques : les hématozoaires des grenouilles et des crapauds de Stanleyville. Annales de Parasitologie, VIII, 1930, p. 127-134.

Wenyon (C.-M.). - Protozoology, London, 1926.

Laboratoire de parasilologie de Stanleyville (Congo belge) 